\title{
Journal of Infectious Pulmonary Diseases
}

Disseminated Tuberculosis in Immunocompetent Patients: Case Reports

\section{Daniel Pereira*, Teresa Sequeira, Sara Rocha, and Betânia Ferreira \\ Internal Medicine resident, Serviço de Medicina, Hospital de Santo António, Centro Hospitalar do Porto, Porto, Portugal}

*Corresponding author: Daniel Pereira, Internal Medicine resident, Serviço de Medicina, Centro Hospitalar do Porto, Largo Prof. Abel Salazar, 4099-001 Porto, Portugal, E-mail: betania_ferreira82@hotmail.com
Received date: 01 Sept 2015; Accepted date: 23 October 2015; Published date: 28 October 2015.

Citation: Pereira D, Sequeira T, Rocha S, Ferreira B (2015) Disseminated Tuberculosis in Immunocompetent Patients, Case Reports. J Infect Pulm Dis 1(1): doi http://dx.doi. org/10.16966/2470-3176.104

Copyright: (c) 2015 Pereira D, et al. This is an open-access article distributed under the terms of the Creative Commons Attribution License, which permits unrestricted use, distribution, and reproduction in any medium, provided the original author and source are credited.

\begin{abstract}
Disseminated tuberculosis is a form of widespread bacilli infection with typical involvement of the lungs and other extra pulmonary organs. It is a rare manifestation of mycobacterial infection and unusual among immune competent individuals.

We present 2 cases of disseminated Mycobacterium tuberculosis infection in two otherwise healthy adults: the first is a 62-year-old man, heavy smoker and with alcohol abuse, who was admitted to our hospital in February 2014. He was being treated for multi sensitive disseminated tuberculosis (lung, lymphatic, vertebral, musculoskeletal, and testicular involvement) since March 2013 with a combination of classic antituberculous agents (isoniazid and rifampicin; had stopped pyrazinamide and ethambutol in August 2013) and was admitted due to vertebral tuberculosis with epidural empyema associated with multiple para vertebral and retroperitoneal tuberculous abscesses, failing to respond to the initial conventional therapy and requiring surgical drainage.

The second refers to a 27-year-old man with no previously known medical disorders, referred to our center in early June 2015 with non-specific constitutional symptoms and acute dry cough, and was diagnosed with disseminated tuberculosis with non-cavitary nodular lung involvement cervical, supraclavicular, mediastinal and retroperitoneal lymphadenitis, pleural and peritoneal effusion and hepatosplenomegaly. A cervical lymph node biopsy was performed, revealing caseating granulomas, with a positive DNA probe followed by confirmatory cultural study for multi sensitive Mycobacterium tuberculosis. The patient is now being treated with a conventional four-drug regimen.

No primary or secondary immunodeficiency was diagnosed following a thorough study during the investigation of these two patients.

Disseminated tuberculosis is a rare and potentially lethal form of tuberculosis that usually affects immune compromised patients. The infection of healthy adults and the occurrence of extra pulmonary involvement is a diagnostic challenge and its poor prognosis can be outfought with the early initiation of anti tuberculous agents and appropriate surgical therapy, when prompted.
\end{abstract}

Keywords: Disseminated tuberculosis; Widespread infection; Healthy adults; Potentially lethal; Anti tuberculous agents

\section{Introduction}

Disseminated Tuberculosis (TB) is a rare form of TB infection characterized by thelymphatic and hematogenous spread of Mycobacterium tuberculosis bacilli. Although the primary site of infection is usually the lung, other organs may be involved either in primary or post-primary infection [1]. In healthy individuals, this dissemination is contained by a prompt immune response, particularly cell-mediated immunity and the interferon (IFN) $\gamma$ pathways, limiting the infection [2]. Therefore, clinical disseminated disease following primary or reactivation of established latent tuberculosis is more often in relatively or absolutely compromised hosts, such as infants, patients with Acquired Immune Deficiency Syndrome (AIDS) or patients with latent infection placed on a tumor necrosis factor alpha blocker, compromising the regular immune surveillance $[3,1]$.

Rarely, TB dissemination occurs in healthy individuals, with no known pre-disposable condition, challenging our clinical suspicion to consider it as a differential diagnosis, especially in the setting of the non-specific clinical features of this form of disease, such as the ones presented in this report.

\section{Case Report 1}

A 62-year-old man, with alcohol abuse and heavy smoker with no know lung disease or history of past infections was being treated for disseminated TB since March 2013. He had been admitted in another hospital and had a report referring multiorgan involvement including pulmonary, lymphatic, testicular, vertebral and paravertebral with bilateral abscesses by a multi sensitive Mycobacterium tuberculosis, and was being treated with a combination of classic anti-tuberculous agents (isoniazid [INH], rifampicin [RIF], pyrazinamide [PZA] and ethambutol [EMB], having stopped the last two after 16 weeks of therapy) with Directly Observed Therapy (DOT). He was Human Immunodeficiency Virus (HIV) seronegative and was on oral prednisolone $(20 \mathrm{mg} /$ day) on the beginning of treatment.

He had chronic lower back pain following the vertebral and paravertebral involvement and was referred to our center in February 2015 following worsening symptoms in the past couple of months, followed by an MRI performed in ambulatory that revealed spondylodiscitis involving the posterior from D12 to L1 and L3-L4 with an anterior spinal epidural empyema along the D9-L4 vertebrae with spinal cord deformation (Figure 1). The MRI also showed multiple bilateral paravertebral abscesses alongside the psoas major muscles, with a massive $130 \mathrm{~mm}$ formation, on the left side.

Initial physical exam revealed vital signs within normal limits and oxygen saturation of $97 \%$ on ambient air. He had pale mucous membranes

Copyright: $\odot 2015$ Pereira D, et al. This is an open-access article distributed under the terms of the Creative Commons Attribution License, which permits unrestricted use, distribution, and reproduction in any medium, provided the original author and source are credited. 
Open Access
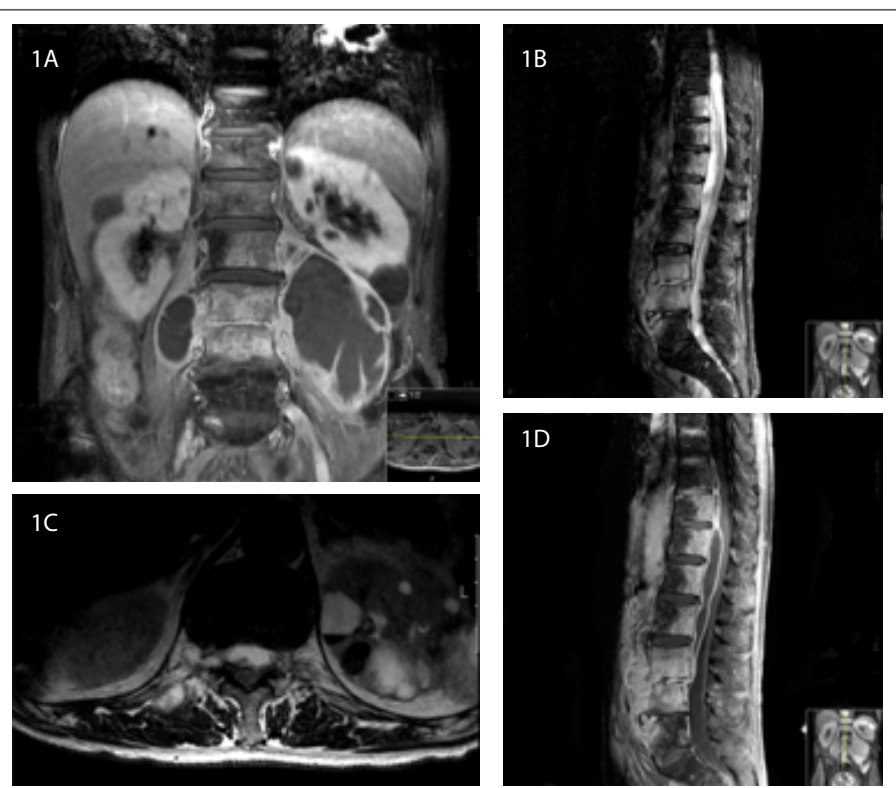

Figure 1: MRI study. 1A) T1 weighted coronal view showing massive paravertebral abscesses, alongside psoas major muscles, as well as enhanced disc, perivertebral soft tissues and vertebral bodies, mainly L3L4 and L4-L5; 1B) Sagittal T2-STIR view with a spinal epidural collection ranging from D10 to $L 4 ; 1 C$ ) T2 weighted axial view showing medullary cone compression at D12-L1 level; 1D) Saggital T1 weighted exhibiting medullary cone compression.

and thoracic and abdominal examinations were unremarkable. He presented with a normal mental status and showed no signs of medullar compression whatsoever. Laboratory test showed normal White Blood Cell (WBC) population count; hemoglobin was $10.0 \mathrm{~g} / \mathrm{dL}$, with normal hepatic transaminases, bilirubin and renal function. The chest CT scan revealed a $43 \mathrm{~mm}$ wide cavitary lesion with an air-fluid level in the lower lobe apical segment of the left lung associated with disperse bilateral small nodular opacities, suggesting a miliary pattern (Figure 2).

He was submitted to percutaneous drainage of the left psoas major abscess, with a positive acid-fast smear and molecular assay with polymerase chain reaction (PCR) for Mycobacterium tuberculosis, although no bacilli were cultured on Lowenstein-Jensen or Middle brook growth mediums. Initial quadruple therapy was maintained with $\mathrm{INH}$, RIF, PZA and EMB.

Failing to achieve clinical improvement, amikacin, moxifloxacin, ethionamide, cycloserin and para-aminosalicylic acid were added to INH, RIF and EMB on the founded clinical suspicion of drug-resistant TB and the patient underwent successful surgical drainage of the paravertebral abscesses on day 20 .

Faint clinical progress was followed by retrosternal chest pain with electrocardiographic and echocardiographic evidence of acute pericarditis with moderate pericardial effusion. A non-steroidal anti-inflammatory drug was initiated with a favorable clinical course, but mild pericardial thickening developed and $1 \mathrm{mg} / \mathrm{Kg} /$ day of prednisolone was introduced to avoid progression to constrictive pericarditis.

Expressive aphasia and right-sided hemi paresis suddenly developed on the 28th day of hospitalization. The cranial CT scan showed just a cortical hyperdense multifocal lesion on

the left frontal, temporal and parietal lobes, suggestive of bacilar tuberculomas, as well as vascular narrowing of the supraclinoid segment of the left internal carotid artery, involving the sphenoidal segment of the middle cerebral artery, producing hipodense lesions in the left lateral post-central gyrus and the frontal semiovale center (Figure 3). These findings alluded ischemic changes due to infectious vasculitis, albeit the patient was already on systemic corticosteroids, meanwhile changed to dexamethasone. A lumbar puncture was performed and meningitis was excluded. A bronchoalveolar lavage was performed, confirming a positive PCR and acid-fast stain for TB. Despite no growth was obtained on cultural studies, molecular assays revealed susceptibility to first-line therapy drugs, INH and RIF, prompting an investigation of alternative causes of treatment failure. He was confirmed to be HIV seronegative, as well as Human T Lymphotropic Vírus (HTLV) 1 and 2. Immunological tests showed normal immunoglobulins and no specific antibodies were detected, including anti-IFN $\gamma$, and no production deficit was detected in this interferon. The patient later developed a severe lymphopenia, with no selective deficiency detected in lymphocyte subsets and no IgG or IgM anti-lymphocyte antibodies were reported. Further investigation revealed no CD119 deficiency and no endoscopic or radiographic findings were suggestive of tuberculous enteritis, confirmed by a normal histology and culture-negative tissue.

Based on the molecular susceptibility pattern, conventional INH, RIF, PZA and EMB therapy was resumed with progressive clinical improvement, elicited by pain amelioration on analgesic drugs, sustained increase in lymphocyte count achieving normal range values and gradual radiologic recovery (Figure 4). Right-sided hemi paresis and motor aphasia persisted and the patient was discharged conducting a rehabilitation program in a convalescence unit, 135 days after he was admitted, on DOT with INH, RIF, PZA, EMB and dexamethasone.

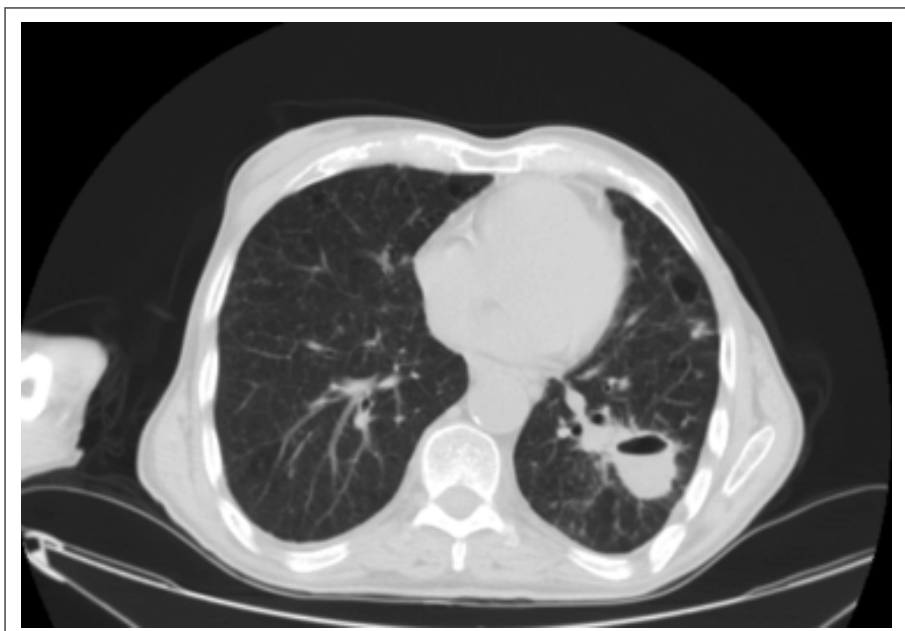

Figure 2: Chest CT scan of the cavitary lesion in the apex of the left lower lobe.
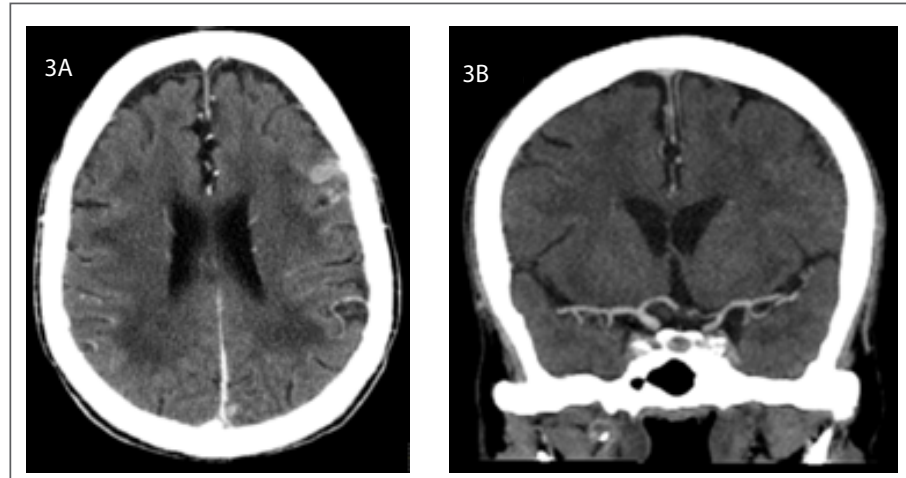

Figure 3: A) Cerebral tuberculoma on an axial view of the brain CT scan; B) Contraste enhanced coronal view showing diffuse narrowing and irregularity of the supraclinoid segment of the left internal carotid artery. 

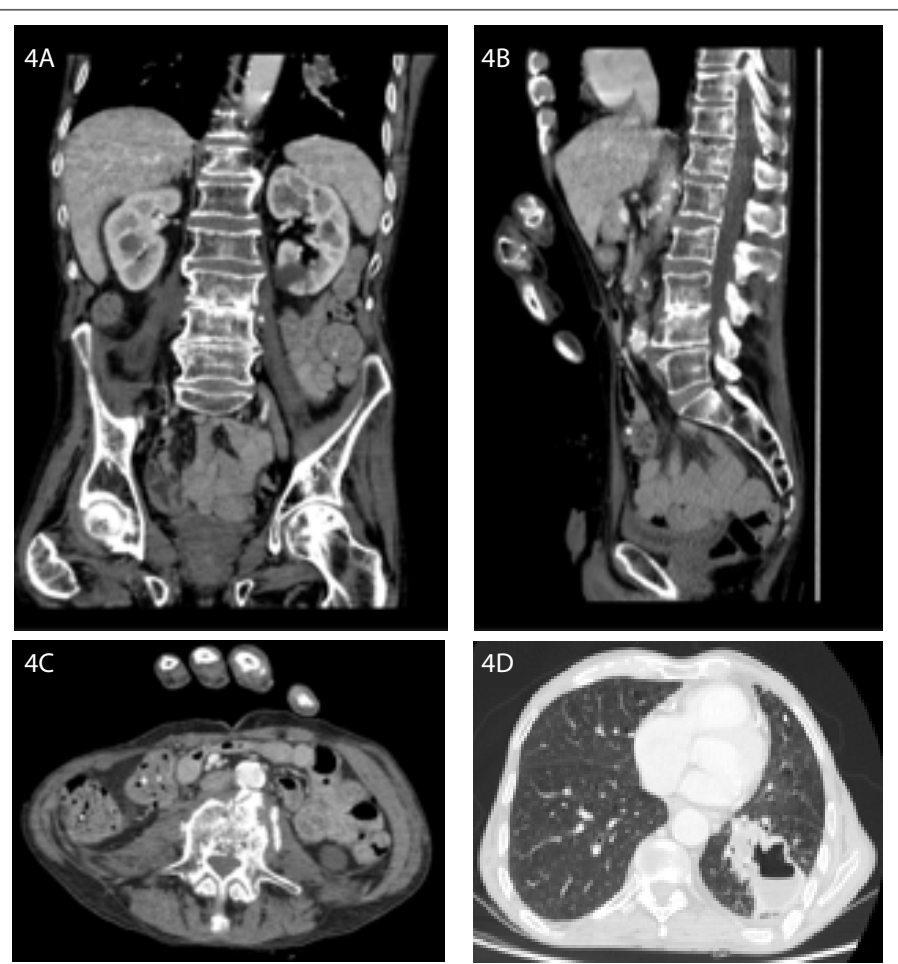

Figure 4: A, B and C. Coronal, sagittal and axial scans of the abdomen and pelvis showing non-enhanced residual lesions in the paravertebral regions and alongside the psoas major muscles, greatly improved when compared to initial MRI images; $D$. Larger volume cavitary lung lesion but peripherally non-contrast enhanced, suggesting abscess in resolution.

Subsequent outpatient management would be guaranteed in the local tuberculosis diagnosis and treatment center.

\section{Case Report 2}

A 27-year-old, previously healthy man was referred to our hospital in May 2015. He had presented with a constitutional syndrome that developed in the past month, which included asthenia, anorexia and weight loss of $15 \%$ of body mass index. Two weeks before admission he started complaining of an unproductive cough and pleuritic chest pain on the lower right hemi thorax. His family physician performed him a chest-abdomen-pelvis CT scan that revealed bilateral apical fibrous scars of the lungs, an imprecise solid nodular area in the right apex, small rightsided pleural effusion, upper Para-aortic lymphadenopathy and small peritoneal effusion. He had been vaccinated for as a child, had no history of symptomatic primary tuberculosis, known contact with infected individuals or recent travel history and was admitted for investigation.

Initial physical examination exhibited normal vital signs and oxygen saturation on ambient air. Non painful right-sided enlarged lateral cervical and supraclavicular lymph nodes were palpable, with a maximum diameter of $20 \mathrm{~mm}$, with a non-adherent elastic consistency. No abnormality was detected on chest and abdomen exploration and no other lymph nodes were apparent.

Basic laboratory tests included a normal WBC count and hemoglobin, renal function, transaminases and bilirubin levels.

A CT scan of the chest, abdomen and pelvis reported the previous fibrotic changes and nodular area in the right upper lobe, added to upper left lobe emphysema with no signs of pleural or pericardial effusion, in addition to numerous conglomerate mediastinal and retroperitoneal lymph nodes with signs of central necrosis, the largest measuring 32 $\mathrm{mm}$ (Figure 5). Mild enlargement of the spleen, liver and pronounced densification of the peritoneum was also observed, with no peritoneal effusion.

Mandatory study included negative consecutive acid-fast stains; Lowenstein-Jensen and Middle brook culture growth mediums of the sputum, followed by a positive Quantiferon-TB and a bronchoalveolar lavage revealing positive acid-fast stain for

Mycobacterium tuberculosis along with cultured bacilli. Excisional cervical lymph node biopsy for histopathologic and microbiologic evaluations exhibited necrotizing granulomatous lymphadenitis, positive molecular assay and cultural growth of a drug-susceptible strain of Mycobacterium tuberculosis.

The patient was confirmed to be HIV seronegative and the following workup for underlying immunodeficiency was negative and included normal immunoglobulin levels, lymphocyte subsets, and specific antibodies.

A conventional 4-drug regimen with INH, RIF, PZA and EMB was initiated and he was discharged for outpatient management.

\section{Discussion}

Disseminated tuberculosis is defined as the widespread form of Mycobacterium infection mostly involving the lungs, lymph nodes, liver, pleura, pericardium, spleen, meninges and bone [4]. It is highly uncommon and it is estimated that disseminated TB accounts for 1 to $2 \%$ of all cases of TB infection in immune competent individuals [5]. 349 cases were reported in the United States in 2012, a minor increase of 1.8\% since 2006 [6].

Commonly known conditions favor extended bacillary disease, such as malnutrition, advanced age, pregnancy, alcohol abuse, corticosteroid or other immunosuppressive and cytotoxic therapy including biologic agents, connective tissue diseases, diabetes mellitus, malignancy, organ transplantation, renal failure and most notably HIV infection, where miliary infection accounts for nearly $10 \%$ of all cases [3,7-10]. Thus, a thorough investigation for primary or secondary immune deficiency is imperative, as it can change the therapeutic approach and global outcome.

Classic presentation is variable and generally includes a sub acute or chronic constitutional syndrome with other absent symptoms or reflecting the underlying organ involved. Can also have a wide-ranging clinical picture, from nocturnal hyperhidrosis, fever of unknown origin or even multi organic failure and, therefore, should always be considered even in the immune competent adult [3].

In general, antimicrobial therapy for disseminated tuberculosis is the same as pulmonary infection although modifications may be warranted in the setting of drug-resistant TB and longer duration of therapy may be appropriate for high organism burden or slow clinical response, immune suppression, CNS infection and in certain patients with bone and joint involvement, where a surgical approach is sometimes mandatory $[11,12]$.

Despite adequate therapy for a drug-susceptible TB strain in the first patient, musculoskeletal lesions failed to improve and he progressed
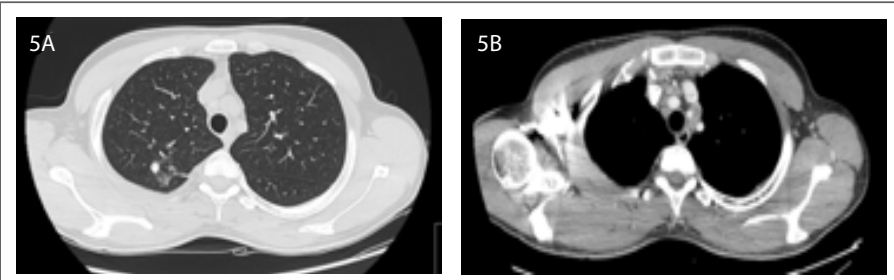

Figure 5: Chest CT scan showing a (A) nodular area in the right upper lobe and $(B)$ numerous mediastinal conglomerates with central necrosis. 
with acute pericarditis and pericardial effusion followed by Central Nervous System (CNS) vasculitis, determining a meticulous immune status workup. Unaffirmative results emerged other questions, such as the insufficient (CNS) penetration of certain anti-tuberculous drugs, like RIF or EMB when compared to moxifloxacin or ethionamide, illustrating the adverse neurological result in this case $[13,14]$.

Another explanation would be the development of a paradoxical Tuberculosis-associated Immune Reconstitution Inflammatory Syndrome (TB-IRIS), with the patient developing signs of disease progression after initial response to treatment. These reactions, largely described in HIV infection, also occur in seronegative individuals and manifestations include recurrent fever, worsening pulmonary infiltrates, pleural or pericardial effusion, new or enlarging tuberculomas and CNS invasion. Pathogenesis of TB-IRIS accounts for the release of new antigen targets during mycobacterial elimination, hypersensitivity to those antigens and excessive immune restoration following bacillary-induced immune suppression in the initial treatment phase [15]. No such workup or hypotheses were conducted for the second patient, as there were no evidences of treatment failure.

Lymphopenia stands for one of the most common laboratory abnormalities in TB-infected patients, in addition to anemia, leucopenia, thrombocytopenia, erythrocyte sedimentation rate, C-reactive protein, hyponatremia or hypocalcaemia, and it constitutes an independent risk factor of mortality [16,17]. It installed progressively in the first patient and prompted an immediate check for underlying causes, which failed to provide a consistent unequivocal response. Considering lymphocyte recovery during anti-tuberculous drug therapy, it was presumed to be secondary to infection via redistribution in secondary lymphoid organs.

Adjunctive systemic glucocorticoid therapy is recommended for treatment of TB involving the CNS and pericardium but data is limited and based on small case reports and scant clinical series $[4,18,19]$. Both forms occurred in the first patient, encouraging steroid therapy, resulting in pericarditis resolution but failed to achieve neurologic recovery, in accordance with the expected morbidity described in literature and establishing it as an independent risk factor of mortality in most studies [20]. Other predictors are pancytopenia or lymphopenia, age, late presentation and serious underlying disease $[16,21,22]$.

\section{Conclusion}

Disseminated tuberculosis is a rare, devastating and potentially fatal form of tuberculosis. It arises from lymphohematogenous spread of the bacilli often resulting in a clinically inapparent condition, but encloses a high morbidity and mortality burden, prompting a high index of suspicion and requiring swift therapeutical action.

Albeit more common in the immune deficient patient, it can also affect healthy individuals, offering a challenging diagnosis in the path of a meticulous investigation.

\section{References}

1. Hilal T, Hurley P, McCormick M (2014) Disseminated tuberculosis with tuberculous meningitis in an immunocompetent host. Oxf Med Case Reports 2014: 125-128.

2. Seneviratne SL, Doffinger R, Macfarlane J, Ceron-Gutierrez L, Amel Kashipaz MR, et al. (2007) Disseminated Mycobacterium tuberculosis infection due to interferon deficiency. Response to replacement therapy. Thorax 62: 97-99.
3. Agu CC, Setu P, Basheer H (2014) Miliary tuberculosis in an immunocompetent male. International Journal of Case Reports and Images 5: 140-144.

4. Lessnau K-D, Cunha BA, de Luise C (2012) Miliary TB. Medspace.

5. Sharma SK, Mohan A, Sharma A, Mitra DK (2005) Miliary tuberculosis: New insights into an old disease. Lancet Infect Dis 5: 415-430.

6. Centers for disease control and prevention (CDC) (2012) Reported tuberculosis in the United States. U.S. Department of Health and Human Services, Atlanta.

7. Braun MM, Coté TR, Rabkin CS (1993) Trends in death with tuberculosis during the AIDS era. JAMA 269: 2865-2868.

8. Aguado JM, Herrero JA, Gavaldá J, Torre-Cisneros J, Blanes M, et al. (1997) Clinical presentation and outcome of tuberculosis in kidney, liver, and heart transplant recipients in Spain. Spanish Transplantation Infection Study Group, GESITRA. Transplantation 63: 1278-1286.

9. Munt PW (1972) Miliary tuberculosis in the chemotherapy era: with a clinical review in 69 American adults. Medicine (Baltimore) 51: 139155.

10. Charfi MR, Dougui MH, Louzir B, Mestiri A, Zbiba M, et al. (1998) Disseminated tuberculosis in non-immunocompromised host: Three case reports. Rev Med Interne 19: 917-920.

11. American Thoracic Society (1986) Medical Section of the American Lung Association. Treatment of tuberculosis and tuberculosis infection in adults and children. Am Rev Respir Dis 134: 355.

12. Oktay MF, Topcu I, Senyigit A, Bilici A, Arslan A, et al. (2006) Followup results in tuberculous cervical lymphadenitis. J Laryngol Otol 120: 129-132.

13. Cherian A, Thomas SV (2011) Central nervous system tuberculosis. Afr Health Sci 11: 116-127.

14. Nau R, Sörgel F, Eiffert $H$ (2010) Penetration of drugs through the blood-cerebrospinal fluid/blood-brain barrier for treatment of central nervous system infections. Clin Microbiol Rev 23: 858-883.

15. Meintjes G, Lawn SD, Scano F, Maartens G, French MA, et al. (2008) Tuberculosis-associated immune reconstitution inflammatory syndrome: case definitions for use in resource-limited settings. Lancet Infect Dis 8: 516-523.

16. Maartens G, Willcox PA, Benatar SR (1990) Miliary tuberculosis: Rapid diagnosis, hematologic abnormalities, and outcome in 109 treated adults. Am J Med 89: 291-296.

17. Isaacs RD, Nicholson GI, Holdaway IM (1987) Miliary tuberculosis with hypercalcaemia and raised vitamin $D$ concentrations. Thorax 42 : 555-556.

18. Dooley DP, Carpenter JL, Rademacher S (1997) Adjunctive corticosteroid therapy for tuberculosis: a critical reappraisal of the literature. Clin Infect Dis 25: 872-887.

19. Centers for Disease Control and Prevention (CDC), American Thoracic Society (2003) Update: adverse event data and revised American Thoracic Society/CDC recommendations against the use of rifampin and pyrazinamide for treatment of latent tuberculosis infection. United States, MMWR Morb Mortal Wkly Rep 52: 735.

20. Kim JH, Langston AA, Gallis HA (1990) Miliary tuberculosis: epidemiology, clinical manifestations, diagnosis, and outcome. Rev Infect Dis 12: 583-590.

21. Hunt BJ, Andrews V, Pettingale KW (1987) The significance of pancytopenia in miliary tuberculosis. Postgrad Med J 63: 801-804.

22. Sahn SA, Neff TA (1974) Miliary tuberculosis. Am J Med 56: 494-505.

Citation: Pereira D, Sequeira T, Rocha S, Ferreira B (2015) Disseminated Tuberculosis in Immunocompetent Patients, Case Reports. J Infect Pulm Dis 1(1): doi http://dx.doi.org/10.16966/2470-3176.104 